Musées, Patrimoine et Culture scientifiques et techniques

$144 \mid 2012$

novembre - décembre 2012

\title{
Vigie-Nature, un réseau de citoyens qui fait avancer la science
}

\section{Romain Julliard}

\section{(Q) OpenEdition \\ Journals}

Édition électronique

URL : http://journals.openedition.org/ocim/1133

DOI : $10.4000 /$ ocim. 1133

ISSN : 2108-646X

Éditeur

OCIM

Édition imprimée

Date de publication : 1 novembre 2012

Pagination : $42-47$

ISSN : 0994-1908

Référence électronique

Romain Julliard, « Vigie-Nature, un réseau de citoyens qui fait avancer la science », La Lettre de I'OCIM

[En ligne], 144 | 2012, mis en ligne le 01 novembre 2014, consulté le 06 mai 2019. URL : http:// journals.openedition.org/ocim/1133; DOI : 10.4000/ocim.1133 
UN OBSERVATOIRE PARTICIPATIF

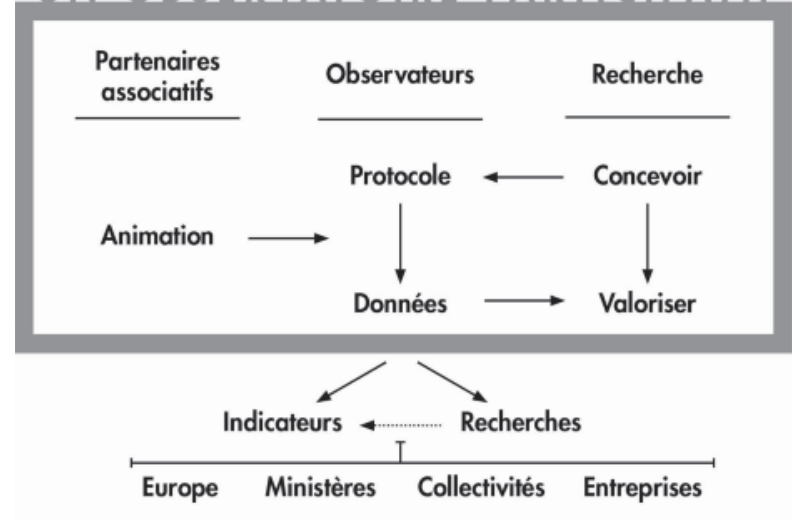

Le fonctionnement repose sur un triple partenariat : - entre le chercheur et l'observateur, le premier doit concevoir un protocole pour le second, en respectant ses motivations, en échange de quoi l'observateur accepte de fournir ses données pour alimenter la recherche : c'est du donnant-donnant; - au niveau de la coordination, entre la recherche et une structure généralement associative en charge de I'animation de la communauté des observateurs; - entre le dispositif de sciences participatives ainsi constitué et un ensemble d'acteurs intéressé par les résultats, qu'ils relèvent de la recherche (amélioration de la connaissance du fonctionnement de la biodiversité) ou de questions plus appliquées comme la production d'indicateurs d'état de la biodiversité. Ces acteurs sont susceptibles de financer le dispositif.

la recherche, il faut donc associer une approche corrélative basée sur l'observation qui permette d'appréhender cette complexité : observations d'assemblages d'espèces répétées sur le long terme et sur de nombreux sites dans des contextes variés. C'est possible en proposant des protocoles simples mais standardisés de recueil des données à un réseau d'observateurs, en s'appuyant sur le volontariat (voir la figure ci-dessus). Ce partenariat entre recherche impliquée dans la conception des plans d'échantillonnage et public connaît un essor important, sous le nom de sciences participatives, au point qu'un auteur nord-américain le qualifie de phénomène typiquement XXIe siècle (Silvertown 2009).

\section{L'état de santé de la nature ordinaire, c'est l'affaire de tous}

Vigie-Nature est un dispositif qui vise à contribuer à combler cette double lacune : coupler un dispositif de collecte de données sur le territoire national à un projet de recherche basé sur cette plateforme. La question de recherche commune à tous les observatoires de Vigie-Nature est de comprendre la réponse de la biodiversité aux changements globaux. Cela posé, la mise en œuvre n'est pas si simple. Comme l'ont bien analysé Cooper et al. (2007), ce genre de dispositif ne fonctionne que si on se trouve à l'intersection de ce qui intéresse le chercheur, ce qui intéresse les observateurs et ce qu'il est faisable d'observer, en fonction des compétences du public visé.

Quand un tel projet rencontre son public, il peut générer des volumes considérables de données : des centaines de milliers de données standardisées d'assemblages d'espèces sont ainsi recensées chaque année en France par les ornithologues amateurs du programme STOC ou par tout un chacun participant à l'Observatoire des Papillons des Jardins. Entre les mains des chercheurs, ces données permettent de tester des prédictions théoriques ou de vérifier le caractère général de processus trouvés dans des études expérimentales ponctuelles (Couvet et al. 2011). On assiste également à un glissement par rapport à la démarche classique de la recherche : au lieu de se focaliser sur ce qu'on sait ne pas savoir, en mettant en œuvre la séquence hypothèseexpérimentation, on met en œuvre un dispositif de collecte de données dont on ne saura qu'une fois constitué à quelles questions il peut répondre. On voit apparaître alors des « surprises », c'est-à-dire, découvrir des phénomènes dont on ignorait même... qu'on les ignorait. Or, ces phénomènes inattendus sont de plus en plus fréquents dans le contexte actuel de changements. Ainsi, ces réseaux d'observations sont les principaux pourvoyeurs de données sur les effets en cours du réchauffement climatique sur la répartition des espèces en les mesurant très précisément : plus intense au nord qu'au sud de l'Europe, plus rapide pour les papillons que pour les oiseaux, mais malgré tout en retard vis-à-vis de l'augmentation des températures... (Devictor et al. 2012). La plupart de ces réseaux ont été mis en place bien avant que le réchauffement climatique ne devienne une réalité illustrant l'une des potentialités majeures de tels observatoires : pourvoir les données pour répondre à nos futures questions.

\section{Sciences participatives: dépasser les vaines querelles}

Le volontariat est souvent associé à une piètre qualité des données récoltées. Or l'expérience montre plutôt l'inverse : un observateur volontaire n'a pas d'autre intérêt à participer que de faire les choses au mieux, si bien qu'à compétence égale, les données issues de volontaires sont en général plus fiables que celles récoltées par des observateurs rémunérés pour le faire. De plus l'intérêt de ces données dépend moins de la fiabilité de chacune que du respect du protocole, qui permet des comparaisons dans le temps et entre sites : c'est la base de données qui est validée statistiquement et pas chacune des données. 


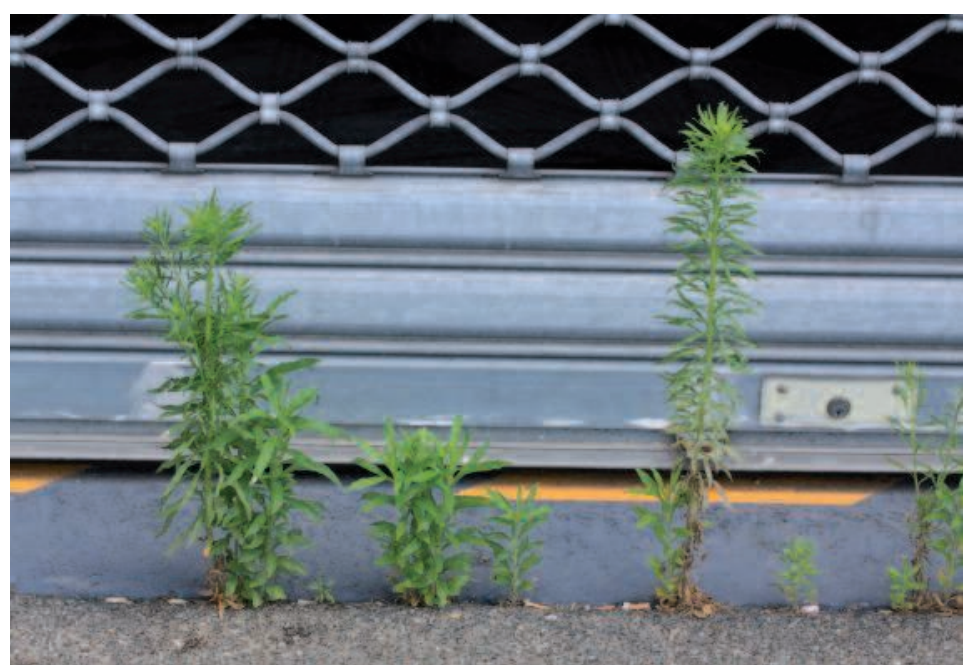

La biodiversité urbaine, "Sauvages de ma rue »: la Vergette du Canada () N. Machon/MNHN

Au sein des communautés scientifiques et naturalistes, ces initiatives ont pourtant presque toujours suscité des controverses. Notons tout d'abord leurs caractères assez franco-françaises : j’ai évoqué cette question avec Jeremy Greenwood (alors directeur du BTO au Royaume-Uni), et il m'a répondu : " mais Romain, ici les scientifiques se battent pour avoir accès aux données de sciences participatives! ». Je vois trois raisons à ces polémiques :
- une incompréhension sur les objectifs : ces suivis s'intéressent à la biodiversité ordinaire, aux espèces communes et ne prétendent pas contribuer à l'étude des espèces rares ;

- une méconnaissance des statistiques qui conduit à la confusion entre biais et précision : bien sûr les observations d'un néophyte sont moins précises que celles d'un spécialiste, mais les observations de 10000 néophytes peuvent être bien plus précises que celles de 100 spécialistes. De plus, le biais (l'écart entre les observations et la réalité) peut être bien plus important dans le cadre des données de spécialistes qui orientent leurs observations vers ce qu'ils connaissent déjà, que chez des observateurs plus naïfs plus enclins à respecter un protocole ;

- "une idée bigote du terme espèce que se font les naturalistes » (pour reprendre les mots de Charles Darwin). Une donnée de biodiversité ne serait «scientifique » que si elle est identifiée « à l'espèce ». Peutêtre est-il bon de rappeler qu'une donnée n'est pas scientifique en soi, c'est l'usage qu'on en fait, les conclusions qu'on en tire qui peuvent l'être ou pas. Pour filer une métaphore bien connue, une pomme qui tombe n'est pas scientifique, ce qui l'est, c'est l'utilisation de cette observation pour illustrer la théorie de la gravité, en laissant la place à la possibilité de la réfuter. Quelle observation est la plus intéressante : « trois papillons blancs dans mon jardin » ou «une Orchidus remarquablus dans une pelouse calcaire
Vigie-Nature est un programme de sciences participatives qui consiste a suivre les especes communes (faune et flore) á léchelle nationale, grâce à des réseaux dobservateurs volontaires.

En s'appuyant sur des protocoles simples et rigoureux, il propose à chacun de contribuer à la recherche en découvrant la biodiversité qui nous entoure. Ainsi, tout le monde peut participer aux observatoires Vigie-Nature, les plus jeunes comme les plus âgés, du débutant au naturaliste confirmé.

Initié il y a plus de 20 ans avec le Suivi Temporel des Oiseaux Communs (STOC) créé en 1989 ,

le programme Vigie-Nature s'est renforcé depuis avec le suivi de nombreux groupes: les papillons, chauves-souris, escargots, insectes pollinisateurs, libellules, plantes.. Dans différents milieux : jardins publics et privés, villes, zones agricoles, zones humides...

En offrant aux scientifiques des données de terrain essentielles, dans toute la France, les observateurs volontaires participent ainsi à l'amélioration des connaissances sur la biodiversité ordinaire et sur ses réponses face aux changements globaux (urbanisation, changement climatique...).

www.vigienature.ff
Pour tous les curieux de nature

6 observatoires

Nul besoin d'être un naturaliste confirme pour s'émerveiller devant la nature. Ces observatoires sont ouverts a tous I Vous en apprendrez beaucoup sur la nature quiv vous entoure et contribuerez a répondre aux a sur ta biodiversite !

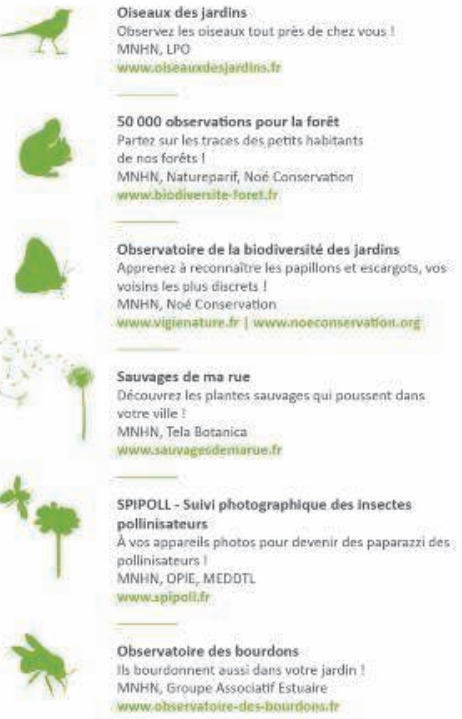

Pour les naturalistes,

6 observatoires

pour aller plus loin

Omithologue, entomologiste, botaniste

ou chiroptèrologue ? Votre contribution

a ces observatoires permettra d'identifitir

les mécanismes des grands changements affectant la biodiversité dans sa globalité et de développer des indicateurs de létat de santé des STOC-Suivi temporel des olseaux commun
Suivi des oiseaux nicheurs au printemps
part leccoute des chants et la copture. Mar THN Nwwingienature.ir > Diseann 5 Stoc

SHOC-Suivi hivernal des oiseaux des champs SHOC-Suivi hivernal des oiseaux des champs

Comptage des o

agricoles.
MNHN, LPO Dròme

Wwiwivigienature. ir > Obeauw > SHOC

(20) Sulvi chauves-souris
Enregistrement des ultasons emis parl les chauves-
souris sur circuits routters ou pedestres.
NNHNN unwowivigionature.frs chatves sonuris

C. Vigie-Flore

2. Inventaire des plantes com MNHN, Tela-sotanica wiwuvingie-1lore,

STEU-Sulvi temporel des libellules Inventaire des libellules dans les zones humides MNHN. CEN NPC, OPIE, SFO www.vigienature.fit > inuectes $>$ sitil STERF - SU France France
Identification et comptage des papillons de jour. MNHN, Association des Lépidopteristes de fronce 
Le SPIPOLL est le Suivi Photographique des Insectes POLLinisateurs. Créé en 2010 par le Muséum national d'Histoire naturelle et l'Office pour les insectes et leur environnement (Opie), les objectifs du SPIPOLLl sont ;

- de mesurer les variations de diversité des insectes pollinisateurs et autres insectes floricoles ;

- d'étudier la macro-écologie des réseaux de pollinisation, sur l'ensemble du territoire métropolitain.

Le SPIPOLL repose sur la participation d'un réseau d'observateurs réalisant un protocole standardisé permettant de collecter des données comparables, à l'échelle de la France. La participation comprend 4 phases :

- la phase « safari-photo » : elle consiste à choisir une espèce de plante en fleur, et à photographier autant que possible les différentes sortes d'insectes la butinant pendant 20 minutes exactement (protocole Flash) ou plus (protocole Long);

- la phase «mise en collection » : trier et recadrer les photos prises lors de la phase précédente afin d'avoir une photo par différent type d'insectes photographiés ; - la phase «identification et envoi des données » : charger les photographies sur le site Internet du SPIPOLL (www.spipoll.org), trouver un nom aussi précis que possible de la plante et des insectes à l'aide d'outils en ligne spécialement conçus pour ce suivi ; - la phase «partage et validation croisée » : sur le Web, chacun peut donner son avis sur les collections et les identifications permettant à tous de progresser dans la convivialité. In fine, un entomologiste de l'Opie valide les identifications.

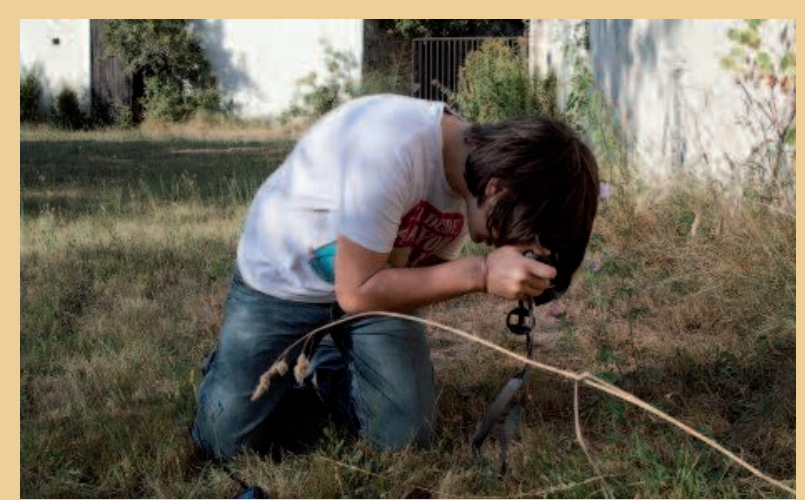

Un observateur du suivi des insectes pollinisateurs ( ) Tangy Lois

À l'issue de ces 4 phases, l'unité d'échantillonnage de base, la « collection », a été constituée : une espèce végétale et un échantillon du cortège d'insectes la visitant, en un lieu et un moment définis. Cette collection ainsi que toutes celles effectuées par le réseau d'observateurs du SPIPOLL sont analysées ensuite au Muséum.

Après deux ans d'existence, le succès est là : des centaines de participants ont déposé plus de 18000 collections (70 000 photographies) et une première publication (Deguines et al. 2012) a consacré l'intérêt scientifique de ce suivi. Le projet est demandeur mais les participants qui réussissent à déposer une collection deviennent rapidement des passionnés. Quel plaisir de découvrir le monde fascinant des insectes de manière à la fois ludique, sérieuse et utile. observée par le spécialiste du genre » ou « une pomme qui tombe »? Cette question n'a pas de sens, ces observations portent une information que l'on peut interpréter scientifiquement (ou pas), et c'est cette interprétation qui sera plus ou moins intéressante.

\section{La biodiversité et le citoyen}

La conservation de la biodiversité est rarement considérée comme du ressort du citoyen. Pourtant, la science participative permet à celui-ci de prendre part de façon individuelle à cette gestion collective, en participant aux relevés des données, à l'étude des résultats, et à la mise en place d'actions de gestion pouvant générer des effets positifs mesurables (Cooper et al. 2007). Les projets de science participative ont ainsi un double objectif : collecter le plus grand nombre de données et sensibiliser en même temps aux enjeux liés à la biodiversité. Ils participent également, nous l'espérons, à l'amélioration de la perception de la science par la société.

Lorsque la connaissance est partagée, cela facilite chez l'individu un sentiment d'appropriation. Or, la gestion collective et partagée de la biodiversité est, ou sera, un atout majeur d'appui aux politiques publiques et à l'évaluation de leur mise en œuvre (Couvet et al. 2008). Les décisions prises en faveur de la biodiversité sont de plus en plus basées sur la connaissance scientifique. Quand cette connaissance est elle-même alimentée par la société civile, on peut s'attendre à ce que cela confère à ces décisions une plus grande légitimité et par là même une meilleure chance d'être mises en œuvre collectivement. Dans un contexte de très forte incertitude (celui du changement global), la capacité d'une société à résoudre des problèmes collectifs peut être améliorée lorsque ses citoyens sont parties prenantes de la science, et un bon moyen d'y parvenir est 
que la problématique et les méthodes scientifiques soient mieux partagées et que les citoyens participent directement aux projets de recherche académique.

\section{0 millions d'observateurs?}

Ce potentiel commence à intéresser deux sphères qui peuvent donner une nouvelle ampleur à ces dispositifs : d'une part à l'initiative du ministère de l'Agriculture et en partenariat avec l'Assemblée Permanente des Chambres d'Agriculture, nous expérimentons la mobilisation d'agriculteurs volontaires dans le suivi de biodiversité dans leurs exploitations (l'Observatoire Agricole de la Biodiversité). Les premiers retours sont très encourageants, les premiers agriculteurs participants (400 en 2012) sont aussi intéressés que les autres publics mais semblent en plus trouver un certain intérêt à montrer qu'il y a effectivement de la biodiversité dans leur exploitation.

\section{Les motivations des observateurs à participer}

Tout responsable de projet de sciences participatives se demande comment mobiliser et fidéliser des observateurs et doit donc s'interroger sur les raisons qui les motivent. Je les classe en 4 catégories, sur une base purement empirique : mon intuition.

La compréhension de l'intérêt scientifique du projet. Ce premier aspect est une affaire de communication de la part de l'animateur scientifique, communication qui malgré tous les efforts semble toujours insuffisante si l'on en croit les participants, avides de retours. C'est un aspect nécessaire (pas de participation si l'intérêt scientifique du projet n'est pas évident) mais malheureusement pas suffisant : presque aucun observateur ne participe que pour la bonne cause, l'avancée de la science ou la gloire du Muséum...

Se sentir nécessaire. Ce deuxième aspect est un écueil sans doute typiquement « latin » : la participation ne semble importante que quand l'observateur se sent indispensable ("personne d'autre que moi ne peut faire cette observation»). Ce facteur est assez compliqué à prendre en compte car il s'oppose à la nécessaire simplicité des protocoles proposés qu'implique un grand nombre d'observateurs aux compétences hétérogènes. Il faut arriver à mobiliser beaucoup de participants en les convainquant qu'ils sont uniques.

Le plaisir à participer. Ce troisième aspect peut sembler évident : la participation doit rester un plaisir (ou au moins offrir une récompense). À nouveau, c'est compliqué car rigueur et simplicité ne vont pas forcément de pair avec plaisir. Un des écueils est que ce plaisir peut être éphémère, par exemple quand il s'agit d'apprendre. C'est très mobilisateur d'apprendre à mettre un nom sur un papillon dans son jardin, mais une fois qu'on sait les reconnaître, il faut offrir autre chose. D'autre part, il faut aussi veiller à ce que cela ne rentre pas en concurrence avec la phase finale de l'observation : la saisie des données. Comme c'est difficile de rendre cette partie ludique, il faut être suffisamment habile pour que la participation ne prenne tout son sens que quand la donnée est déposée, en jouant par exemple sur l'argument que tant que la donnée n'est pas saisie, la participation est vaine.

Appartenir à un réseau social. Ce dernier aspect est sans doute celui qui a été le plus ignoré dans la jeune histoire de Vigie-Nature. La science participative est une activité collective et le participant de facto appartient à une communauté d'observateurs qui font en même temps la même chose. Ce sentiment d'appartenance, de lien social est sans doute très important pour nombre d'observateurs et donc pour leur fidélisation. Pour l'instant, nous utilisons des outils assez primitifs pour renforcer ce sentiment de communauté : newsletter, forum, site Web éditorial riche en information... Tout indique cependant que c'est une approche de court terme et que les observateurs ont tendance à se lasser. De plus, le recrutement de nouveaux observateurs semble essentiellement dépendre de notre communication et pratiquement pas du bouche-à-oreille (dit « communication interpersonnelle »). Dans un futur proche, il faut sans doute renverser cette logique et construire une communauté à qui on propose de participer : notre richesse, ce ne sont pas les données, mais les observateurs.

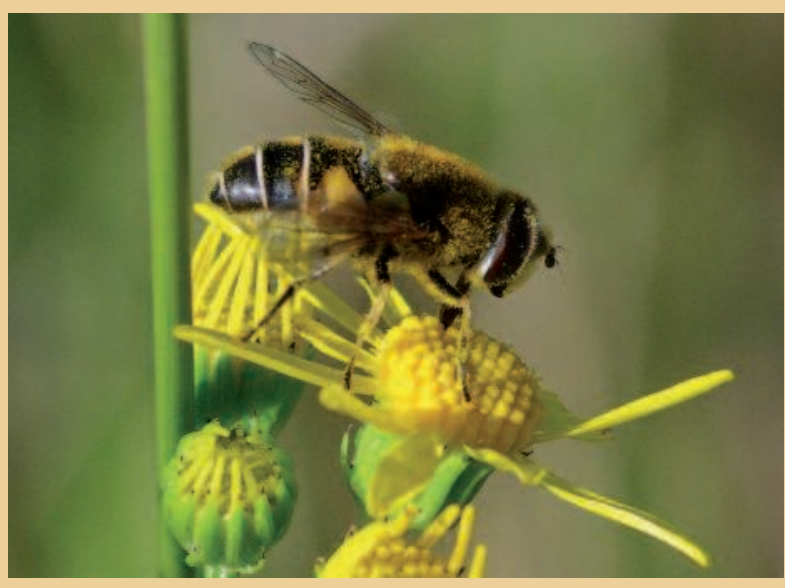

Les insectes pollinisateurs : I'Abeille (c) Nicco, observateur Spipoll 
Plus ambitieux mais moins inattendu, à la demande de l'Éducation nationale, nous avons mobilisé des enseignants du collège et du lycée pour adapter certains de nos protocoles aux programmes scolaires en respectant deux principes : l'offre doit être une façon de mettre en œuvre les programmes existants (et ne pas s'y ajouter) ; les parcours proposés doivent permettre une participation «pour de vrai » au projet VigieNature. De manière très significative, c'est la démarche scientifique plus que l'éducation à la biodiversité qui séduit ici le ministère. Les premiers pas sont à nouveau extrêmement encourageants.

Il ne faudrait pas croire pour autant que la science participative en général et Vigie-Nature en particulier ont atteint leur apogée : c'est nouveau, et donc immature et imparfait, mais offre de nombreuses perspectives pour être notamment, plus participatif, plus local, plus expérimental, plus pertinent par rapport aux enjeux liés à la biodiversité. Les bases de données collectées ont un potentiel qui intéresse malgré tout de plus en plus de scientifiques et, on peut attendre beaucoup d'innovation notamment d'ordre statistique, ces bases sont des mines d'or peu explorées. La science participative doit continuer à faire ses preuves mais je parie qu'elle a de beaux jours devant elle et que nous ne faisons aujourd'hui qu'entrevoir ses potentialités.

\section{Bibliographie}

Cooper, C.-B., Dickinson, J., Philips, T. et Bonney R. Citizen science as a tool for conservation in residential ecosystems, Ecology and Society, $\mathrm{n}^{\circ} 11,2007$.

Couvet, D., Jiguet, F., Julliard, R., Levrel, H. et Teyssedre, A. Enhancing citizen contributions to biodiversity science and public policy, Interdisciplinary Science Reviews, n³3, 2008, pp. 95-102.

Couvet, D., Devictor, V., Jiguet, F. et Julliard R. Scientific contributions of extensive biodiversity monitoring. Comptes rendus Biologies. 2011, pp. 370377.

Deguines, N., Julliard, R., De Flores, M. et Fontaine, C. The whereabouts of flower visitors : contrasting land-use preferences revealed by a countrywide survey based on citizen science, PLoS ONE, 2012.

Devictor, V., Van Swaay, C., Brereton, T., Brotons, L., Chamberlain, D., Heliölä, J., Herrando, S., Julliard, R., Kuussaari, M., Lindström, Å., Reif, J., Roy, D.-B., Schweiger, O., Settele, J., Stefanescu, C., Van Strien, A., Van Turnhout, C., Vermouzek, Z., Wallis DeVries, M., Wynhoff, I. et Jiguet, F. Differences in the climatic debts of birds and butterflies at a continental scale, Nature Climate Change, n², 2012, pp. 121-124.

Silvertown, J. A new dawn for citizen science. Trends in Ecology and Evolution, n²4, 2009, pp. 467-471. 